\title{
Bilateral upper-limb rehabilitation after stroke using a movement-based game controller
}

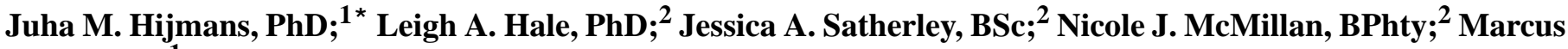 \\ J. King, $\mathrm{BE}^{1}$ \\ ${ }^{1}$ Industrial Research Ltd, Christchurch, New Zealand; ${ }^{2}$ REAL Neurological Research Group, Centre for Physiotherapy \\ Research, University of Otago, Dunedin, New Zealand
}

\begin{abstract}
This study aimed to determine the effectiveness of a bilateral, self-supported, upper-limb rehabilitation intervention using a movement-based game controller for people with chronic stroke. Fourteen participants received a control treatment, followed by a washout period, and then the intervention. The intervention comprised playing computer games with the CyWee Z (CyWee Group Ltd; Taipei, Taiwan), a movement-based game controller similar to the Nintendo Wii remote. The CyWee $\mathrm{Z}$ was incorporated into a handlebar, making bilateral exercises possible by allowing the unaffected side to support and assist the affected side. The intervention lasted for 8 to 10 sessions of 45 to 60 minutes over a period of 2.5 weeks. The Fugl-Meyer Assessment upper-limb section (FMA-UL) was used as the primary outcome. The Wolf Motor Function Test and the Disabilities of Arm, Shoulder, and Hand outcome measure were used as secondary outcomes. Postintervention, motor performance as measured by the FMA-UL was significantly improved compared with all preintervention assessments $(p<0.001)$, whereas no changes were found on both secondary outcomes. It can be concluded from this pilot study that upper-limb motor performance of adults with chronic stroke improves with repetitive, game-assisted, selfsupported bilateral exercises.
\end{abstract}

Key words: bilateral therapy, computer gaming, intervention, motor performance, outcomes, rehabilitation, self-assisted, selfsupported, stroke, upper limb.

\section{INTRODUCTION}

An estimated 16 million people per year worldwide experience a stroke, of which about two-thirds survive [1].
Six months poststroke, 30 to 66 percent of them show motor deficit of the arm contralateral to the lesion [2]. Activity-based rehabilitation can improve upper-limb motor function [3]; this is of great importance, because lack of arm-movement control directly affects activities of daily living and independence [4].

To improve motor performance, both motor (re)learning and compensatory strategies are required [5-6]. Motor (re)learning and recovery are mainly possible because of plasticity of the brain [7], and the changes caused by plasticity in the lesioned hemisphere coincide with motor function improvement after activity-based rehabilitation [3]. In addition to neural plasticity changes in the lesioned side, motor recovery may occur because of a shift of balance in the motor cortical recruitment toward the undamaged hemisphere via the ipsilateral pathways [8-9].

Movement practice and repetition form the basis of plasticity-based motor recovery [10-14]. A number of factors are thought to enhance plasticity-based rehabilitation,

Abbreviations: $\mathrm{CI}=$ confidence interval; $\mathrm{DASH}=$ Disabilities of Arm, Shoulder, and Hand outcome measure; FMA-UL = Fugl-Meyer Assessment upper-limb section; $\mathrm{PC}=$ personal computer; WMFT = Wolf Motor Function Test.

*Address all correspondence to Juha M. Hijmans, PhD; Department of Rehabilitation Medicine, Center for Rehabilitation, UMCG, PO Box 30.001, 9700 RB Groningen, the Netherlands; +31-50-3610108; fax: +31-50-3611708.

Email: juha hijmans@hotmail.com

DOI:10.1682/JRRD.2010.06.0109 
such as task-oriented movement practice in a challenging, engaging, functional, and meaningful way, and the rehabilitation should address body function and structures, activity, and participation level [9-13,15]. Rehabilitation has shown that, even in chronic stroke, improved upperlimb outcomes can be achieved [16-17].

Recovery after stroke correlates with the frequency and intensity of exercise [11,13,18-19]; however, passive movement is insufficient to alter motor recovery [19]. Active engagement and movement attempts are thought to be more important than passive movement, and the focus should be on movement coordination rather than muscle strengthening [19].

Moreover, strong evidence exists that bilateral training is effective in functional recovery of the upper limb [2021]. Summers et al. suggest that bilateral synchronous movement therapy is more effective than similar unilateral training [22]. Bilateral therapy is based on the idea that involvement of the unaffected upper limb facilitates learning the spatial and temporal parameters required for motor recovery of the affected limb [21]. Bilateral training is thought to increase activation of the affected hemisphere, especially the secondary motor areas, by interhemispheric connections [21-22].

Technology-assisted upper-limb training after stroke can provide engaging and task-oriented training in a natural environment using patient-tailored feedback to support (re)learning of motor skills [9]. Computer and video games can improve therapy compliance through engagement [13]. Gaming consoles like the Nintendo Wii (Nintendo; Redmond, Washington) and the Sony Eyetoy (Sony Computer Entertainment, Inc; Tokyo, Japan) are currently used in upper-limb stroke rehabilitation, although limited clinical evidence exists for their effectiveness [23-24]. Console games are designed for nondisabled people; therefore, they are often too fast for people with motor disabilities to use [25] and frequently provide negative feedback when a game is lost.

Robotic therapy has been reported to improve several motor control aspects (e.g., muscle-activation pattern, selectivity, and speed) and may have long-term effects [26]. Robot-assisted therapy appears to improve motor control more than conventional therapy and is suited for rehabilitation in both the acute and subacute phase [26]. However, the advantages probably exist only because a higher intensity of practice can be reached [13,27], resulting in improvement at a body function and structures level and not necessarily improvement in activities of daily living
$[13,26]$. A disadvantage of robotic therapy is the cost; thus, an important question regarding the future of robotic rehabilitation is whether similar objectives can be accomplished by a simpler and more cost-effective approach [28-29].

We have developed a system that uses low-cost gaming technology to exercise the affected upper limb of people with stroke while their less-affected arm supports and assists the movements of the affected arm in a bilateral manner. In this pilot study, we investigate the effects of this system on motor recovery, which is designed to provide repetitive, game-assisted upper-limb rehabilitation in adults with chronic stroke.

\section{METHODS}

\section{Participants}

Adult participants at least 6 months poststroke were recruited from the local community by media advertising. Exclusion criteria comprised no voluntary arm movement, self-reported problems preventing device use (orthopaedic, medical, and/or painful conditions), and an inability to understand the project and its requirements.

\section{Study Design}

In a control-washout-intervention design, the participants served as their own controls. Participants were assessed after being enrolled in the study (T0 = precontrol). During the initial control period, participants played four simple mouse-based computer games on a personal computer (PC) with their unaffected arm: Solitaire, Mah-Jong, FreeCell, and Bejeweled. Three participants played on three PCs with one or two therapists supervising during a 2.5 week period of 8 to 10 sessions, each lasting between 45 and 60 minutes. The participants were retested after the control treatment $(\mathrm{T} 1=$ postcontrol $)$ and again after 2 to 3 weeks ( $\mathrm{T} 2=$ preintervention) during which no intervention was provided. Finally, participants received the intervention, followed by the last assessment (T3 = postintervention).

The intervention comprised playing games on a PC using a CyWee Z game controller (CyWee Group Ltd; Taipei, Taiwan) incorporated into a custom-made handlebar (Figure 1). As in the control treatment, three participants played on three PCs with one or two therapists supervising, the same amount of therapist interaction provided during the control treatment. A suite of games provided a graduated series of physical challenges, from strategic, stationary target-hitting games (Bejeweled and Balloon Popping), 


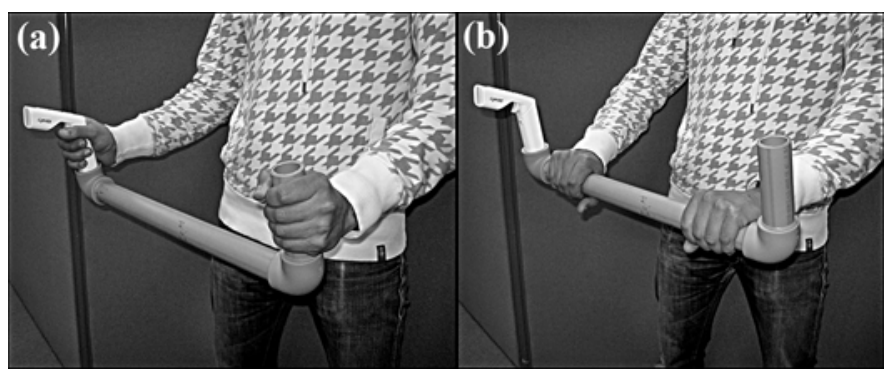

Figure 1.

CyWee Z game controller (CyWee Group Ltd; Taipei, Taiwan) incorporated into handlebar. (a) "Hands vertical" for exercising radial and ulnar deviation (combined with elbow and shoulder flexion/ extension). (b) "Hands horizontal" for exercising wrist flexion/ extension (combined with elbow and shoulder flexion/extension).

to moving target-hitting games (Mosquito Swat, Music Catch, and ReBounce) and faster sports games (10-Pin Bowling and Air Hockey), as well as some casual games (Paint By Numbers) and puzzle games (Mah-Jong and Solitaire). All games required large cursor movements in both the horizontal and vertical direction. In the first two sessions, the therapist presented three simple target-hitting games (Mosquito Swat, Music Catch, and ReBounce). In the following sessions, the participants could choose which game to play-either a new or a known game. The cognitive requirements to play the games were low, because the games were simple target-hitting games with large, easy-tosee objects; basic sports games; or simple puzzle games, including the well-known Solitaire and Mah-Jong. Most games were specifically developed or adapted for people with stroke so that graphics were clear and fast reaction speed was not required. A therapist would advise the participant to choose an easier game if he or she was not able to cope with the cognitive requirements of a certain game. Movement was the main objective of this study; the games were used to guide movements and motivate the participant to move.

During each session, the participants made an estimated minimum of 500 repetitions and possibly up to 800 repetitions. One repetition could be an up-down movement, a left-right movement, or (in most cases) a combination of both (e.g., moving cursor from center to top-right corner of screen).

If the participants were able to use the CyWee $\mathrm{Z}$ trigger button with their affected hand, the CyWee $Z$ was placed in that hand ( $n=11$ ); otherwise, the CyWee $Z$ was placed in the unaffected hand. If grip strength was insuf- ficient to hold the game controller, a therapist fixed it to the participant's wrist with a soft bandage $(n=2)$.

\section{Device}

The CyWee $\mathrm{Z}$ is a movement-based game controller similar to the Nintendo Wii controller. It was incorporated into a handlebar between 350 and $500 \mathrm{~mm}$ long (Figure 1). Rotations of the device in the transverse plane produced horizontal mouse cursor translations on the screen, while rotations in the sagittal plane produced vertical mouse cursor translations (Figure 2).

The handlebar could be held two ways (Figure 1): (1) "hands vertical" for exercising radial and ulnar deviation (vertical mouse cursor translations) combined with elbow (vertical and horizontal mouse cursor translations) and shoulder flexion and extension (horizontal mouse cursor translations), and (2) "hands horizontal" (pronated) for exercising wrist extension and flexion (vertical mouse cursor translations) combined with elbow (vertical and horizontal mouse cursor translations) and shoulder flexion and extension (horizontal mouse cursor translations). When hands were held horizontal, only non-mouse-clicking games could be played. All games required both horizontal and vertical mouse cursor translations. Therefore, wrist, elbow, and shoulder movement of both the affected and the unaffected sides were required to play. The therapists instructed participants to practice with both hands vertical and hands horizontal and observed every session.

\section{Outcome Measures and Statistics}

During all assessment sessions, therapists performed the Fugl-Meyer Assessment upper-limb section (FMAUL) [30]; the Wolf Motor Function Test (WMFT) [31]; and the Disabilities of Arm, Shoulder, and Hand outcome measure (DASH) [32-33]. We used the FMA-UL as the primary outcome, whereas we considered the WMFT and the DASH secondary outcomes. We tested the effects of the intervention using an analysis of variance for repeated measures ( $\alpha=0.05$ for all statistical tests). Significant within-subject effects were Bonferroni adjusted in multiple comparison tests. We used MedCalc version 11 (2009) for all statistical analyses. We recorded the actual time spent on game play and on rest periods within the sessions during sessions 3 and 7 . 


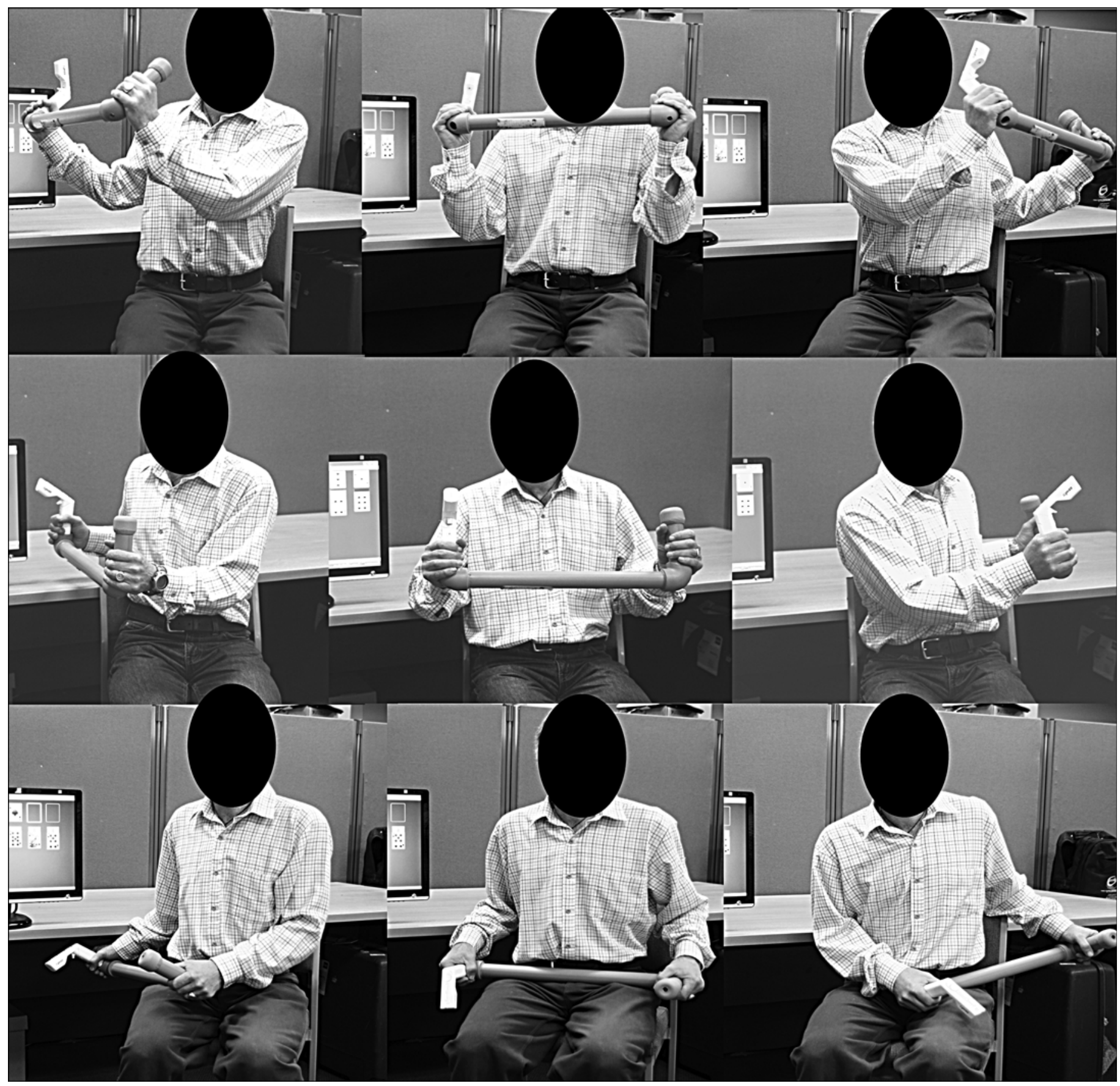

Figure 2.

Movements required for controlling cursor on computer screen using CyWee Z game controller (CyWee Group Ltd; Taipei, Taiwan) incorporated into handlebar.

\section{RESULTS}

Twenty-two adults poststroke were recruited, of which 16 were eligible for this study. One person dropped out before the start of the trial and another dropped out before the intervention because of family commitments. Five women and nine men with an average age of $71 \pm 12$ (mean \pm standard deviation; range: 47-85) completed the 
trial. Eight participants had had a stroke in their left hemisphere and six in their right. One person was left-arm dominant prestroke with the left arm affected by the stroke; so, in nine participants the dominant hand was affected and in five the nondominant. The control therapy was played with the unaffected hand (5 dominant and 9 nondominant). Eleven participants used the trigger of the CyWee $\mathrm{Z}$ with their affected hand. Three participants had insufficient grip to use the trigger with their affected hand; therefore, they used the CyWee $\mathrm{Z}$ with their unaffected hand. Time since stroke was between 1 and 6 years. The mean FMA-UL score at inclusion was $44.2 \pm 17.9$ and ranged from 14 to 65 (maximum score: 66).

The Table shows the outcomes at T0, T1, T2, and T3. A significant within-subject session effect was found on the primary outcome measure, the FMA-UL ( $p<0.001)$. In a multiple comparison test (Bonferroni corrected), the FMA-UL score at T3 was significantly higher than at T0, $\mathrm{T} 1$, and $\mathrm{T} 2$, with an average increase and 95 percent confidence interval (CI) of 5.00 (CI: 1.45-8.55; $p=0.005$ ), 5.23 (CI: 1.80-8.66; $p=0.003$ ), and 4.23 (CI: 1.81-6.66; $p=$ 0.001 ), respectively.

No significant within-subject or multiple comparison effects were found on the WMFT and the DASH (Table). Of the 14 participants who completed the trial, one missed the T1 assessment; therefore, we did not take the outcomes of this participant into account in the statistical analyses. When we performed the same statistical analyses on T0, T2, and T3 $(n=14)$, the same outcomes remained significant (data not presented).

Average session time increased from 49.3 to $52.1 \mathrm{~min}$ utes between intervention sessions 3 and 7. The average cumulative rest time during sessions decreased from 3.6 to 1.0 minutes, meaning that the actual game-play time increased by 11 percent between sessions 3 and 7 .

\section{DISCUSSION}

This is the first study conducted using the CyWee $\mathrm{Z}$ as an upper-limb rehabilitation tool and using self-assisted bilateral movements with a movement-based game controller. This pilot study showed that repetitive, bilateral game-assisted exercises using the CyWee $\mathrm{Z}$ incorporated into a handlebar improved upper-limb motor performance measured by the FMA-UL. Even though the group comprised adults with chronic stroke, the cohort was heterogeneous (FMA-UL scores ranged from 14 to 65 at inclusion), and even though the intervention had only 8 to 10 sessions, the intervention improved motor performance significantly. The mean improvement as an effect of the intervention ranged from 4.2 (compared with T2) to 5.2 (compared with T1). These results are comparable with the more intensive robotic-aided therapies reported by Prange et al., with FMA-UL score improvements between 3.4 and 6.1 [26]. Others have also shown that a highly repetitive, synchronous bilateral intervention over a short duration, albeit with no mechanical or computer device included, can be effective [22].

We found no effects on the WMFT and the DASH; the possible explanation for this is that the intervention focused on repetitive arm movements and not on functional or participatory arm activities. The DASH, a selfreport questionnaire, assesses upper-limb function at an activity and participation level, whereas the FMA-UL assesses the upper limb on the body function and structures level [34]. The WMFT is a timed, performancebased measure that measures the speed of performing motor tasks. It can take time for people to incorporate body function and structure improvement and increased task-performance speed, and because assessment was carried out immediately after a relatively short intervention, the WMFT may not be responsive enough to measure change in such a time frame. The low intensity of the

Table.

Mean \pm standard deviation of outcome measures precontrol (T0), postcontrol (T1), preintervention (T2), and postintervention (T3).

\begin{tabular}{lcccccc}
\hline $\begin{array}{l}\text { Outcome } \\
\text { Measure }\end{array}$ & T0 & T1 & T2 & T3 & F & p-Value \\
\hline FMA-UL & $44.2 \pm 17.9$ & $44.0 \pm 17.2$ & $45.0 \pm 16.2$ & $49.2 \pm 16.6$ & 10.41 \\
WMFT & $32.7 \pm 50.4$ & $32.4 \pm 50.6$ & $31.5 \pm 50.9$ & $30.5 \pm 51.2$ & 2.74 & 0.06 \\
DASH & $51.8 \pm 21.5$ & $54.5 \pm 23.4$ & $55.0 \pm 24.4$ & $55.6 \pm 23.2$ & 0.66 & 0.58 \\
\hline
\end{tabular}

${ }^{*} n=13$.

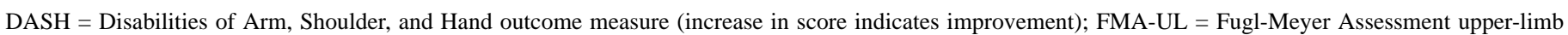
section (increase in score indicates improvement); WMFT = Wolf Motor Function Test (decrease in score indicates improvement). 
intervention compared with constraint-induced therapy could be another explanation for the lack of change measured by the WMFT. Additionally, the WMFT includes movement tasks more frequently performed by the dominant hand but does not consider dominance.

We incorporated the CyWee $\mathrm{Z}$ into a handlebar for several reasons. First, the unaffected side can support and assist the affected side in a bilateral manner by using the handlebar. Bilateral therapy has proven to be effective in functional recovery of the upper limb [20-21]. Second, rotation of the CyWee $\mathrm{Z}$ results in synchronous bilateral gross motor movements with the handlebar, whereas while the CyWee $\mathrm{Z}$ is held in one hand without the handlebar, only limited wrist movement is needed to control the cursor. Third, the handlebar can be placed on the lap as a support against gravity yet still enable gross motor movement if the user has difficulty lifting the device. In their review of bilateral therapy, Latimer et al. suggest that bilateral training provides the requirements considered necessary for motor relearning and focuses on the importance of active involvement of the affected side rather than the affected hand being driven by a robot or the unaffected side [21]. Bilateral training using the CyWee $\mathrm{Z}$ requires this active involvement of the affected upper limb.

The CyWee $\mathrm{Z}$ is a movement-based game controller similar to the Nintendo Wii but coupled to a PC. Therefore, it is possible to develop and use games paced to suit a user's ability level. This system provides a platform for self-assisted, engaging, highly repetitive, and task-oriented bilateral training of the upper limb, all modalities crucial for motor recovery following stroke [13,27]. Previously, expensive robotic therapy was seen as the only way to deliver such a therapy [29].

Overall, game play appeared to be engaging and acceptable for participants. A range of games was provided that had various levels of challenge, and participants selected games according to their own ability and preference. All participants were able to use the equipment. Some participants played only the cognitively easiest games and frequently needed the therapist's help to manage the PC; others played all games, including the more challenging sports games, without assistance. A possibility for improvement could be performance-driven games, where the patient's performance dictates future session targets [34], ensuring that each participant works at an appropriate level.
This pilot trial had limitations. The research design used was a pre-baseline-post design in which the participants were observed over time. It was not possible to blind the assessors, and no control group was recruited. In this study, all participants received the control treatment first, followed by the intervention. In general, a design in which 50 percent of the participants receive the intervention first and 50 percent receive the control treatment first would be a preferable approach. We chose not to use this approach, because if natural recovery would still take place, more recovery would be expected sooner poststroke (during the first weeks of the study). In our design, more natural recovery was expected during the control therapy. Therefore, it was possible to demonstrate that the intervention showed more improvement than natural recovery in combination with a control intervention. We controlled for therapist-interaction effect by using a control treatment consisting of the same amount of therapistinteraction time as during the actual intervention. No differences were found between the control treatment and the washout period. Therefore, therapist-interaction time appeared not to bias our results. Only the immediate effects were studied; a follow-up study should be performed to study the long-term effects of the intervention.

Four participants mentioned shoulder pain during and after the intervention. This pain did not prevent them from participating but should be considered when the game controller is used more frequently, for a longer duration, or without supervision. The first few sessions appeared fatiguing for most participants, reflected by the shorter session lengths and longer break times in session 3 than in session 7 . While quantity, duration, and intensity are important variables in (re)learning motor skills and changing neural structure [35], these results suggest that the start of an upper-limb rehabilitation program may need a ramping regime to slowly increase quantity, duration, and intensity to ensure that sessions are pain free.

\section{CONCLUSIONS}

This study showed that computer-assisted, bilateral upper-limb rehabilitation using a low-cost game controller can significantly improve upper-limb function over a short duration in adults with chronic stroke. Engaging in computer games using a CyWee $\mathrm{Z}$ incorporated into a handlebar seems to be a cost-effective alternative to other forms of therapy, such as robotic, catering toward repetitive, 
self-supported upper-limb exercises. Therefore, based on this study, performing a larger-scale controlled trial with a longer intervention duration and follow-up is recommended. We expect further improvements when the game controller is used over increasingly longer sessions with a longer intervention period.

\section{ACKNOWLEDGMENTS}

\section{Author Contributions:}

Study concept and design: M. J. King, J. M. Hijmans.

Acquisition of data: J. A. Satherley, N. J. McMillan.

Analysis and interpretation of data: J. M. Hijmans.

Drafting of manuscript: J. M. Hijmans.

Critical revision of manuscript for important intellectual content:

L. A. Hale, M. J. King.

Statistical analysis: J. M. Hijmans.

Obtained funding: M. J. King.

Administrative, technical, or material support: M. J. King.

Study supervision: L. A. Hale.

Financial Disclosures: The authors have declared that no competing interests exist.

Funding/Support: This material was based on work supported by the Foundation for Research Science and Technology, New Zealand (contract CO8X0816).

Additional Contributions: The authors thank the participants, who willingly gave their time and ideas during intervention trial, and Cerebralfix, New Zealand, for supplying the majority of the computer games. Dr. Hijmans is now with the Department of Rehabilitation Medicine, Center for Rehabilitation, University Medical Center Groningen, Groningen, the Netherlands.

Institutional Review: All participants signed informed consent forms and the study was approved by the University of Otago Human Ethics Committee (09/193).

Participant Follow-Up: The participants have already been informed of the intent to publish. Authors do not intend to provide the details of publication to participants directly; they will be informed through public access to the academic Web site.

\section{REFERENCES}

1. Strong K, Mathers C, Bonita R. Preventing stroke: Saving lives around the world. Lancet Neurol. 2007;6(2):182-87. [PMID: 17239805] DOI:10.1016/S1474-4422(07)70031-5

2. Kwakkel G, Kollen BJ, Wagenaar RC. Therapy impact on functional recovery in stroke rehabilitation: A critical review of the literature. Physiotherapy. 1999;85(7):377-91. DOI:10.1016/S0031-9406(05)67198-2

3. Richards LG, Stewart KC, Woodbury ML, Senesac C, Cauraugh JH. Movement-dependent stroke recovery: A system- atic review and meta-analysis of TMS and fMRI evidence. Neuropsychologia. 2008;46(1):3-11. [PMID: 17904594]

DOI:10.1016/j.neuropsychologia.2007.08.013

4. Whitall J, McCombe Waller S, Silver KH, Macko RF. Repetitive bilateral arm training with rhythmic auditory cueing improves motor function in chronic hemiparetic stroke. Stroke. 2000;31:2390-95. [PMID: 11022069]

5. Langhorne P, Coupar F, Pollock A. Motor recovery after stroke: A systematic review. Lancet Neurol. 2009;8(8): 741-54. [PMID: 19608100$]$ DOI:10.1016/S1474-4422(09)70150-4

6. Krakauer JW. Motor learning: Its relevance to stroke recovery and neurorehabilitation. Curr Opin Neurol. 2006;19(1): 84-90. [PMID: 16415682] DOI:10.1097/01.wco.0000200544.29915.cC

7. Hallett M. Plasticity of the human motor cortex and recovery from stroke. Brain Res Rev. 2001;36(2-3):169-74.

[PMID: 11690613$]$

DOI:10.1016/S0165-0173(01)00092-3

8. Jang SH. A review of the ipsilateral motor pathway as a recovery mechanism in patients with stroke. NeuroRehabilitation. 2009;24(4):315-20. [PMID: 19597268]

9. Timmermans AA, Seelen HA, Willmann RD, Kingma H. Technology-assisted training of arm-hand skills in stroke: Concepts on reacquisition of motor control and therapist guidelines for rehabilitation technology design. J Neuroeng Rehabil. 2009;6:1. [PMID: 19154570]

DOI:10.1186/1743-0003-6-1

10. Krouchev NI, Kalaska JF. Virtual worlds and games for rehabilitation and research. Virtual Rehabil. 2008:113-20.

11. Cameirão MS, Badia SB, Verschure PF. Virtual reality based upper extremity rehabilitation following stroke: A review. J Cyber Ther Rehabil. 2008;1(1):63-74.

12. Sveistrup H. Motor rehabilitation using virtual reality. J Neuroeng Rehabil. 2004;1(1):10. [PMID: 15679945]

DOI:10.1186/1743-0003-1-10

13. Kwakkel G, Kollen BJ, Krebs HI. Effects of robot-assisted therapy on upper limb recovery after stroke: A systematic review. Neurorehabil Neural Repair. 2007;22(2):111-21.

[PMID: 17875068] DOI:10.1177/1545968307305457

14. Woldag H, Hummelsheim H. Evidence-based physiotherapeutic concepts for improving arm and hand function in stroke patients: A review. J Neurol. 2002;249(5):518-28.

[PMID: 12021939] DOI:10.1007/s004150200058

15. World Health Organization. International classification of functioning, disability, and health: ICF. Geneva (Switzerland): WHO Publishing; 2011.

16. Van der Lee LH, Wagenaar RC, Lankhorst GJ, Vogelaar TW, Deville WL, Bouter LM. Forced use of the upper extremity in 
chronic stroke patients: Results from a single-blind randomized clinical trial. Stroke. 1999;30(11):2369-75. [PMID: 10548673]

17. Page SJ, Gater DR, Bach-Y-Rita P. Reconsidering the motor recovery plateau in stroke rehabilitation. Arch Phys Med Rehabil. 2004;85(8):1377-81. [PMID: 15295770] DOI:10.1016/j.apmr.2003.12.031

18. Henderson A, Korner-Bitensky N, Levin M. Virtual reality in stroke rehabilitation: A systematic review of its effectiveness for upper limb motor recovery. Top Stroke Rehabil. 2007; 14(2):52-61. [PMID: 17517575] DOI:10.1310/tsr1402-52

19. Krebs HI, Volpe B, Hogan N. A working model of stroke recovery from rehabilitation robotics practitioners. J Neuroeng Rehabil. 2009;25(6):6. [PMID: 19243615] DOI:10.1186/1743-0003-6-6

20. Cauraugh JH, Lodha N, Naik SK, Summers JJ. Bilateral movement training and stroke motor recovery progress: A structured review and meta-analysis. Hum Mov Sci. 2010; 29(5):853-70. [PMID: 19926154]

DOI:10.1016/j.humov.2009.09.004

21. Latimer CP, Keeling J, Lin B, Henderson M, Hale LA. The impact of bilateral therapy on upper limb function after chronic stroke: A systematic review. Disabil Rehabil. 2010; 32(15):1221-31. [PMID: 20156045] DOI:10.3109/09638280903483877

22. Summers JJ, Kagerer FA, Garry MI, Hiraga CY, Loftus A, Cauraugh JH. Bilateral and unilateral movement training on upper limb function in chronic stroke patients: A TMS study. J Neurol Sci. 2007;252(1):76-82. [PMID: 17134723] DOI:10.1016/j.jns.2006.10.011

23. Yavuzer G, Senel A, Atay MB, Stam HJ. "Playstation EyeToy games” improve upper extremity-related motor functioning in subacute stroke: A randomized controlled clinical trial. Eur J Phys Rehabil Med. 2008;44(3):237-44. [PMID: 18469735]

24. Saposnik G, Mamdani M, Bayley M, Thorpe KE, Hall J, Cohen LG, Teasell R; EVREST Study Group for the Stroke Outcome Research Canada Working Group. Effectiveness of Virtual Reality Exercises in STroke Rehabilitation (EVREST): Rationale, design, and protocol of a pilot randomized clinical trial assessing the Wii gaming system. Int J Stroke. 2010;5(1):47-51. [PMID: 20088994] DOI:10.1111/j.1747-4949.2009.00404.X

25. Grammenos D, Savidis A, Georgalis Y, Stephanidis C. Access invaders: Developing a universally accessible action game. Computers Helping People with Special Needs, Proceedings of the 10th International Conference, ICCHP; 2006 Jul 12-14; Linz, Austria. Berlin Heidelberg (Germany): Springer; 2006. p. 388-95.
26. Prange GB, Jannink MJ, Groothuis-Oudshoorn CG, Hermens HJ, IJzerman MJ. Systematic review of the effect of robot-aided therapy on recovery of the hemiparetic arm after stroke. J Rehabil Res Dev. 2006;43(2):171-84.

[PMID: 16847784]

DOI:10.1682/JRRD.2005.04.0076

27. Mehrholz J, Platz T, Kugler J, Pohl M. Electromechanical and robot-assisted arm training for improving arm function and activities of daily living after stroke. Cochrane Database Syst Rev. 2008;(4):CD006876. [PMID: 18843735]

28. Johnson MJ, Feng X, Johnson LM, Winters JM. Potential of a suite of robot/computer-assisted motivating systems for personalized, home-based, stroke rehabilitation. J Neuroeng Rehabil. 2007;4:6. [PMID: 17331243]

DOI:10.1186/1743-0003-4-6

29. Lum PS, Burgar CG, Van der Loos M, Shor PC, Majmumdar M, Yap R. MIME robotic device for upper-limb neurorehabilitation in subacute stroke subjects: A follow-up study. J Rehabil Res Dev. 2006;43(5):631-42.

[PMID: 17123204] DOI:10.1682/JRRD.2005.02.0044

30. Fugl-Meyer AR, Jääskö L, Leyman I, Olsson S, Steglind S. The poststroke hemiplegic patient. 1. A method for evaluation of physical performance. Scand J Rehabil Med. 1975; 7(1):13-31. [PMID: 1135616]

31. Wolf SL, Catlin PA, Ellis M, Archer AL, Morgan B, Piacentino A. Assessing Wolf Motor Function Test as outcome measure for research in patients after stroke. Stroke. 2001; 32(7):1635-39. [PMID: 11441212]

32. Hudak PL, Amadio PC, Bombardier C. Development of an upper extremity outcome measure: The DASH (Disabilities of the Arm, Shoulder and Hand) [corrected]. The Upper Extremity Collaborative Group (UECG). Am J Ind Med. 1996;29(6):602-8. [PMID: 8773720]

DOI:10.1002/(SICI)1097-0274(199606)29:6<602::AIDAJIM4>3.0.CO;2-L

33. Beaton DE, Davis AM, Hudak P, McConnell S. The DASH (Disabilities of the Arm, Shoulder and Hand) outcome measure: What do we know about it now? Brit J Hand Ther. 2001;6(4):109-18.

34. Metcalf C, Adams J, Burridge J, Yule V, Chappell P. A review of clinical upper limb assessments within the framework of the WHO ICF. Musculoskeletal Care. 2007;5(3): 160-73. [PMID: 17610309]

DOI:10.1002/msc.108

35. Jack D, Boian R, Merians AS, Tremaine M, Burdea GC, Adamovich SV, Recce M, Poizner H. Virtual realityenhanced stroke rehabilitation. IEEE Trans Neural Syst Rehabil Eng. 2001;9(3):308-18. [PMID: 11561668] DOI:10.1109/7333.948460 
Submitted for publication June 2, 2010. Accepted in revised form March 7, 2011.

This article and any supplementary material should be cited as follows:

Hijmans JM, Hale LA, Satherley JA, McMillan NJ, King MJ. Bilateral upper-limb rehabilitation after stroke using a movement-based game controller. J Rehabil Res Dev. 2011;48(8):1005-14.

DOI:10.1682/JRRD.2010.06.0109

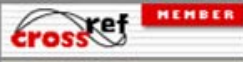

crossaer.oRG 
